\title{
RF BASED TALKING SigNAgE FOR BLIND NAVIGATION
}

\author{
Aswathy V R ${ }^{1}$, Dilraj. $N^{2}$,Sethuraman Rao ${ }^{3}$ \\ Amrita Center for Wireless Networks and Applications, Amrita Vishwa Vidyapeetham, \\ Amritapuri, Kollam, India
}

\begin{abstract}
The major challenge of visually impaired person is in mobility, object identification and identification of space around him/her. The proposed RF Based Talking Signage for Blind Navigation aims to provide a universal electronic travel guide for the visually challenged people. This system incorporates a user friendly and versatile method called "Talking Signage" that is implemented using android devices. The system uses an Android application in the mobile phone which could deliver voice messages about the user environment via a heterogeneous network. It can be deployed in any dense environment so that blind persons can fulfill their needs. The primary advantage of the system compared to other system in the area is low cost, ease of transport, less power consumption, lightweight, and it could be utilized by those peoples who are technically challenged. The architecture proposed in this paper clearly shows communication between a mobile phone and a heterogeneous network enabled with RF devices. We have implemented the system in our university environment and the proposed system found to be a great success.
\end{abstract}

\section{KEYWORDS}

RFdevice;Android app;Visually impaired; Indoor navigation; nesC language

\section{INTRODUCTION}

According to World Health Organization 285 million people are visually impaired worldwide: 39 million are blind and 246 million have low vision due to various reasons [2]. Blindness makes their life despondent because they are not able to pursue a good education since study materials available in Braille are very expensive. Mobility and travel is highly visually oriented with signage in ink print. There is no possibility that this information can be provided to a blind person in Braille. If there is a device which could deliver information about the user environment then it could assist them to move around independently, therefore they could achieve many things in their life which is not feasible at present.

There are systems which are using video sequence learning algorithm and RFID technologies; both are very costly, heavy weight, inconvenient to carry, not circumstantial and need internet accessibility always. This makes blind people to keep away from using assistive technology, refer Figure 1 . So here we are proposing a low cost, easy to carry, less power consuming, lightweight system that could deliver voice messages through mobile phone about the user environment. Hence the proposed system solves above mentioned problems to a great extent. 


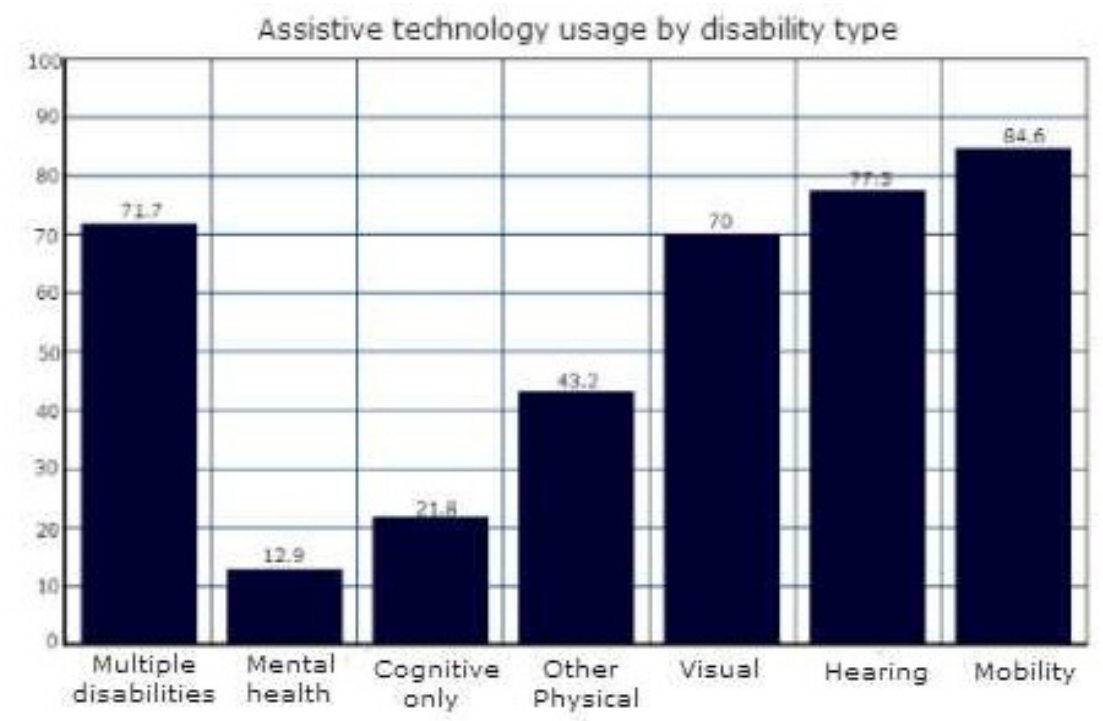

Figure 1. Assistive technology usage:statistics [1]

The main components in the system are a waist belt consisting of Micaz mote, mda100, Blue link Silver Bluetooth module and an Android mobile phone. The system uses Micaz mote which is placed in the location for identification and another in the waist belt of the user. The motes will communicate through Zigbee and the information will be handed over to the mobile through Bluetooth via Blue link silver Bluetooth module. The background application running inside the phone will identify the information came from Bluetooth as a unique id and the id is now cross checked with the database in the mobile. Information which is saved in the database in the mobile phone for the given id will be sent to the text to speech module and is now played through the mobile. Thus the visually challenged will be able to identify the object and spaces around him/her accurately.

The remaining part of this paper is organized as follows: section II describes related works in the area of blind navigation in indoor and outdoor environment. Detailed system architecture is illustrated in section III. Section IV discusses about the algorithms developed. Section V explains the results and discussions. Section VI concludes with future research ideas.

\section{RELATED WORK}

The article [3] describes about a navigation tool called smart walking stick for visually challenged person. The framework can be utilized to catch deterrents, pit, water before visually impaired, and discovering area so that route challenges of visually impaired can be lessened. At present blind people are commonly using white cane which works on the basis of tactile-force feedback or guide dogs. The problems with white cane are it could detect only solid obstacles, heavier, can't be folded and its range is very limited. In the case of guide dog, it is very costly and reacts only to those situations in which it was trained.

The smart stick is equipped with sensors to give information about the environment. Different components used in this system are ultrasonic sensor, water sensor, pit sensor, GPS receiver, level converter, driver, vibrator, voice synthesizer, keypad, speaker, pic controller and battery. GPS technology is integrated with pre-programmed locations in the memory to determine the optimal route to be taken. Microcontroller stores the data of the current location that it receives from the GPS. Then its compared with the destination location that user entered by keypad. 
International Journal on Cybernetics \& Informatics (IJCI) Vol. 4, No. 2, April 2015

Using this information, the system can trace out the distance from the destination and produce alarm to alert the user in advance. Ultrasonic sensors could detect objects whose width or diameter is not less than $3 \mathrm{~cm}$.

The result of experiments conducted with this technology shows that the water sensor could detect presence of water only if the water level crosses $0.5 \mathrm{~cm}$. That is the stick must be dipped in the water. The experiment is done only by storing four destination places in the memory. Limitations of this system are the following.

- This system could not be used for indoor navigation.

- The person could travel to only those places which are stored in the memory.

- The water sensor could detect the water by dipping stick into it.

- It is unable to find out locations or spaces inside building.

- It is not telling the speed of approaching vehicle or obstacle.

- Need high memory for route information storage.

- If satellite signals are weak then the person could not find out his location.

The system RF based talking signage for visually challenged is entirely different from this system, no methods or ideas are similar to that used in Smart walking stick.

Article [4] by Sakmongkon, Chumkamon presents a system using RFID technology to provide location information as well as information about shortest path to the destination .This system is proposed mainly to overcome the inability of GPS and also to reduce the power consumption of components in other navigation and location identification technology.

The RFID tags that contain RFID id and tag location will embed into the footpath where the blind used to walk. The RFID reader in user cane will read it, along with this information; user will input the destination place and pass it into MCU. Using GPRS technology, the information will pass into the server. After receiving it, server will calculate the shortest distance between current location and destination and send back to MCU. The MCU will send this information into MMC (multimedia card memory).From there the information is passed into a speaker. Thus the user comes to know about his/her location and route to destination place. Energy saving is done by activating RFID tag only in the presence of a tag reader.

The assumptions here are that the problem with delay and system size doesn't affect the user even though it is affecting the overall performance. Other disadvantages of the system are the following.

- The RFID tags must be embedded all along the foot path where the blind used to walk.

-GPRS technology is affected with cold start cycle problem.

- It will take time to access the information from a MMC

- GPRS can't be used because of the cost factor involved.

- For embedding along the footpath, 1000s of RFID tags will be required.

- Delay is high.

- Server needs to store bulk amount of data.

- Prone to server failure.

- Connectivity problem.

- Tag and reader must be at close proximity.

These issues can be explained to some degree by utilizing the proposed system. Idea of sending id to a server and taking information from there using GPRS technology is modified in this application by sending id to mobile phone and taking information from database inside the mobile 
phone. Thus problems related to delay, connectivity, etc. are solved, and there is no cost for sending data.

In another work [5] tells about a system which helps blind for navigation. It consists of vibrators, ultrasonic sensors mounted on shoulders of user, and sensors mounted on cane. The system will provide information about distance of overhanging obstacle in real time. It could detect obstacle from 0.03 to 6 meters and operates by sending an ultrasound pulse. This pulse will be reflected from an obstacle in the path. From the time difference between the outgoing transmitted pulse and its echo being received, distance is calculated. The information is then relayed to the blind in some vibro tactile way and speech way for obstacle detection.

Another method is using micromap. It has two modes of operation record and playback. In the record mode the user will store all the information about his routes and when he turns right he will also store the right turn instruction in the microcontroller memory. In the playback mode, the aid measures again the distance travelled by the user and if it is the same as that of the stored distance then it indicates the user to take a right turn [6].

This system is proposed to overcome the problem in other navigation technologies, which are not able to detect the overhanging obstacle. The problems in this system are

- Obstacle detection is not done in hemispherical approach.

- Person using this system is not able to identify different spaces inside a building. Using this system it could identify only the routes and obstacle.

- Since the system is using large number of sensors, energy consumption is high.

- The person has to enter the name of the destination through keypad. This will be difficult for a blind person.

- Large amount of memory is needed.

Paper [7] presents a system for blind which could help in independent mobility in an unknown environment without any manual assistance. Features of the system are object recognition, $\log$ records of all users tag access, user track information and emergency button. In this system user has a RFID reader module with a microcontroller attached into the cane, Zigbee transceiver for transmitting the tags information and TTS for playing information to the user. On the server side Zigbee transceiver is used for wireless communication.

In this technique, RFID passive tag is deployed on the path for object recognition in the house or building. RFID tags will be installed in the floor of the pathway. The RFID reader in the blind cane will read the id from the tag on the object or in the floor and passed to the server using Zigbee. Server will resend the information corresponding to the Id to the RC8660FP TTS module where the received text is translated into speech and informed to the user via head phone. This system also contains an emergency button. When user presses the button, information will be sent to the administrator of the server. The administrator tracks the user by using last retrieved information from the server. Thus he could send manual help for blind person when he is in trouble [6].

System is experimented with 30 tags to form a grid over the path. Out of 30 Tags, 20 tags contain Tag ID and its path location details and remaining10 tags contain Tag ID and attached objects details. All the tags are placed at the path location and at the objects. After placing the tags VIAS database is updated with Tag ID and details associated with each Tag[8].

The proposed system, RF Based Talking Signage for Visually Challenged is not using sensors. The system is able to identify different spaces inside the building and able to provide all 
information about the location which are prerecorded. The system is easy to carry since it is implemented using a mobile phone, a belt attached with motes and its supporting elements which can be worn at the side of the waist belt. It is also not using any GPS technology. The system is of low cost since we are using the users' mobile phone to implement the application. In future this system can be extended for outdoor use. Storage memory is not restricted, we are able to store maximum amount of information that is needed for blind. Zigbee communication technology is used between transmitter and receiver rather than Bluetooth communication to avoid pairing problems. Since it is using the database inside the phone, it's not affected with connectivity problem or server failure or no delay will occur. System is using Bluetooth to get information from the mote which is attached to the location. The RFID mote using will be embedded into a location wherever the user need to identify, like coffee shop, escalator area, class rooms, office rooms etc. Communication range can be extended up to $30 \mathrm{~m}$, according to the user need. In talking signage system no sensors are used and there is no need to give any input to the system. The system will automatically send information about the current location and deliver a voice message about the location.

\section{ARCHITECTURE AND DESIGN}

The proposed system consists of wall mounted RF transmitter, a user module in the waist belt and an application installed user's Android mobile phone. The minimum requirements for Android are a $200 \mathrm{MHz}$ processor, 32MB RAM, and 32MB of storage.

\subsection{User module}

The user module consists of receiver RF device, Bluetooth module [10], and MDA 100 [11].The use case diagram of the system is shown below refer Figure 2.

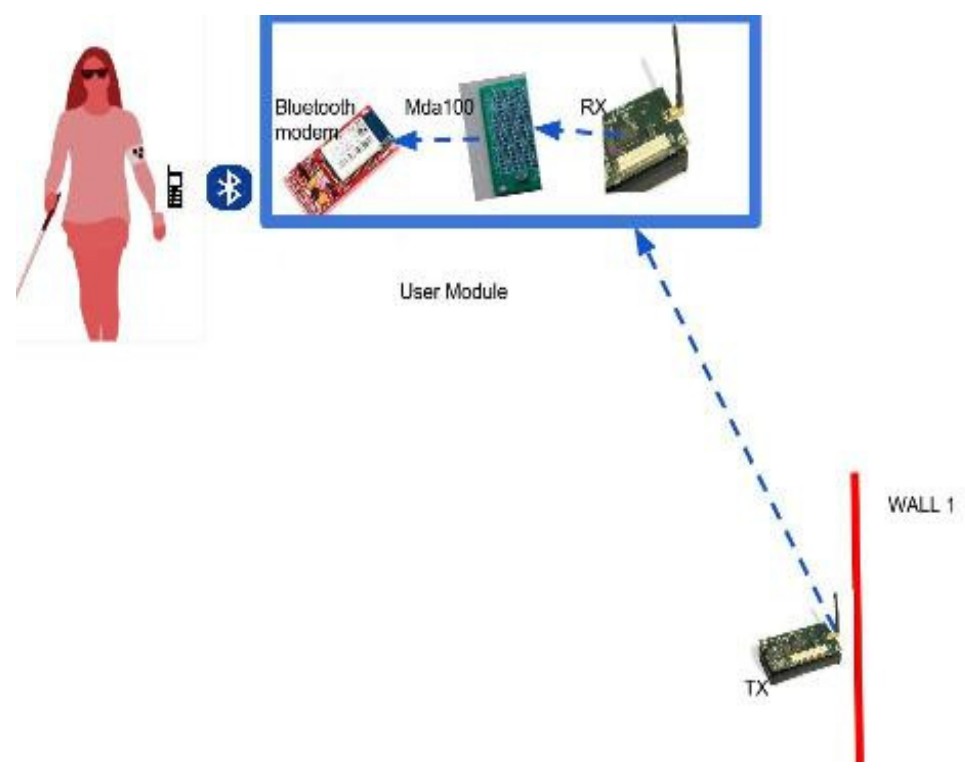

Figure 2. Use case diagram

The receiver RF device used here is a Micaz mote [9]. It is programmed to receive beacons from the RF transmitter mote. Here the transmission power is adjusted to 3milliVolt to avoid interference with adjacent RF modules. The detailed components interaction diagram is shown below refer Figure 3 
International Journal on Cybernetics \& Informatics (IJCI) Vol. 4, No. 2, April 2015

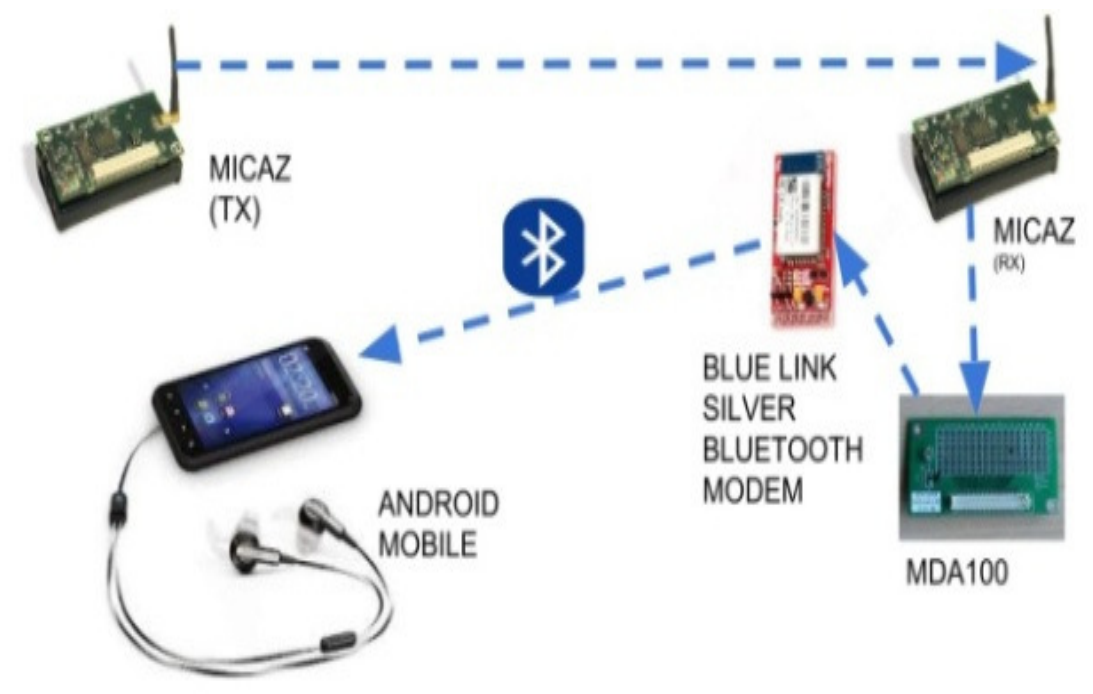

Figure 3. Devices communication

In order to translate Zigbee information into Bluetooth information a Bluetooth module called Blue link silver Bluetooth module is used.

\subsubsection{Blue Link Silver Bluetooth Module}

In order to built a communication between Bluetooth module and the receiver mote the baud rate must be same, so the mote is programmed into a baud rate of $115200 \mathrm{bps}$. The module is shown in the figure 4.

\subsubsection{Specifications}

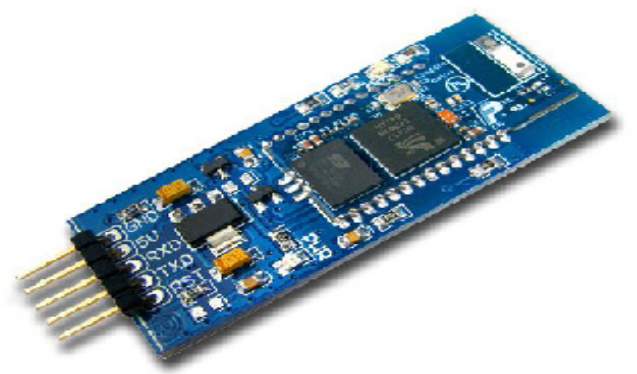

Figure 4. Blulink Silver Bluetooth Module

- It acts as an UART.

- 3V Power supply

- Baud rate 115200.

- 5 pins are Vcc, GND, TX, RX, Reset

- Bluetooth core V 2.0 compliant 


\subsection{MDA 100}

It is used to make an interface between Micaz and Bluetooth module refer Figure 5. It act as a data acquisition board.

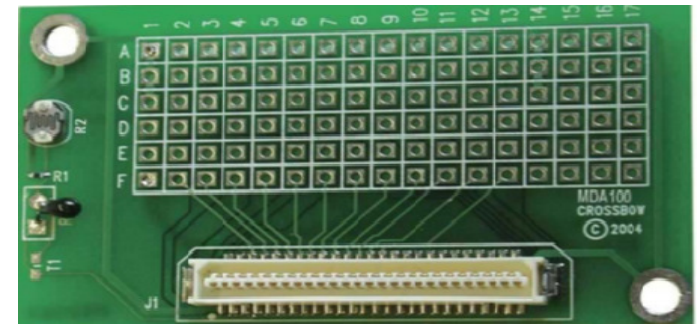

Figure 5. MDA 100

\section{Algorithm For Transmitter And Receiver}

The language used to program the mote is nesC in Linux platform. The algorithm used for transmitter and receiver are the following.

\subsection{Transmitter}

Step1: Start the radio.

Step2: Start the timer

Step3: When the timer fired, create the packet and set node id.

Step4: Set the power.

Step5: Broadcast the packet.

Step 6: If successfully sent toggle led.

\subsection{Receiver}

Step 1: Adjust the baud rate so that it could communicate with the Bluetooth module.

Step2: Start the radio and Std control

Step3: Extract the payload and check whether the received id is needed and sent to the UART

\subsection{Android application}

Step1: Get the Bluetooth Adapter. It is required for all Bluetooth activity.

Step 2: Call the method isEnabled() to find the other Bluetooth enabled devices.

Step 3 : getBondedDevices () .To see already bonded devices

Step 4: call the method startActivityForResult(Intent, int) to make the device discoverable Step 5: For connecting devices, one will be a server by using BluetoothServerSocket.

The server socket will listen for incoming connection requests.

Step 6: BluetoothSocket help to share data between devices.

Step 7: Read data from UART

Step 8: Check if data is needed

Step 9: Create a popup view. 
Step10: Set background images and other animations

Step11: Send data to Text to speech module

\subsection{Flow chart explaining working of the system}

Flowchart of the working system is shown in figure 6.

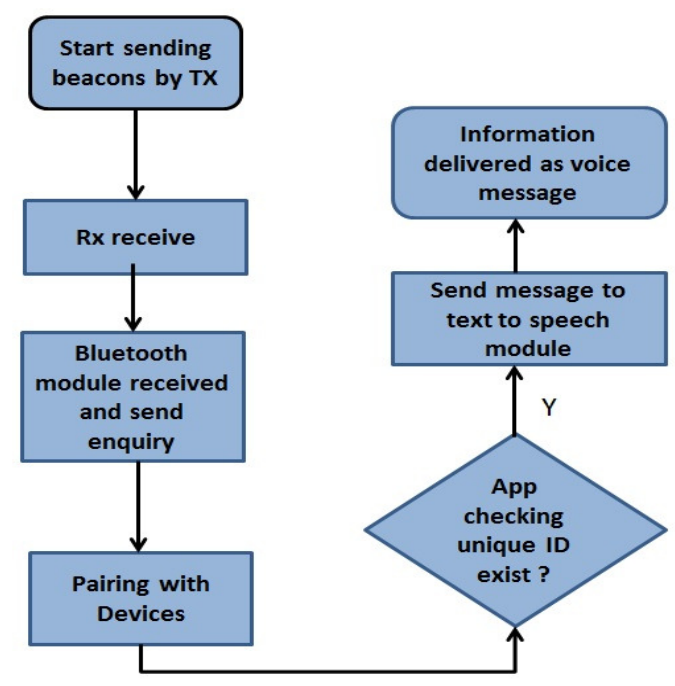

Figure 6.Flowchart of System

\section{RESULTS AND DISCUSSION}

As previously noted, the purpose of this research work was to build an RF based blind navigation system for indoor navigation. The application developed will search for a Bluetooth enabled Blue Link Silver module in the user module. When accept for connection is given, it will begin to receive data from the Bluetooth module. The GUI of the developed application having background of floor plan is shown below refer Figure 7.

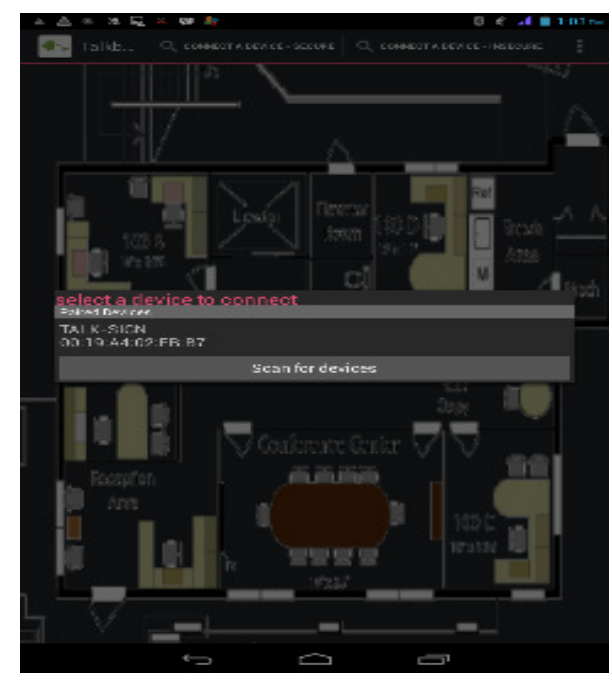

Figure 7. Scan for device 
The first figure shows the paring up with Bluetooth module. The window shows that a unique id ' 1 ' is received from the user module. This number represents that the person is in front of communications lab. For convenience a unique id is given to represent similar areas of a building refer Figure 8.For example, all wash rooms are provided with the same id.

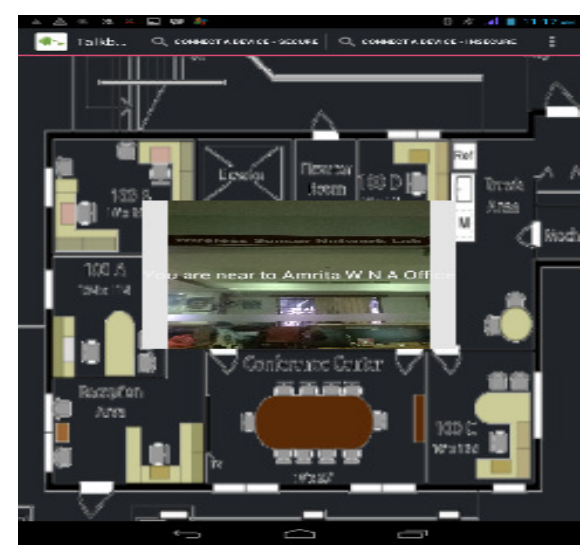

Figure 8. Located place

Bluetooth module is paired with the Bluetooth in the mobile phone. The background talking signage application retrieves the corresponding message for the unique id received and sends it to text to speech module in the phone. The message is now delivered as voice message .Thus the user is able to locate his/her current place. The complete experimental setup is shown in Figure 9.Two micaz motes are used as transmitters and the one which is attached with bluetooth module will act as receiver. The tab shown in figure will receive information from the receiver mote and deliver voice message.

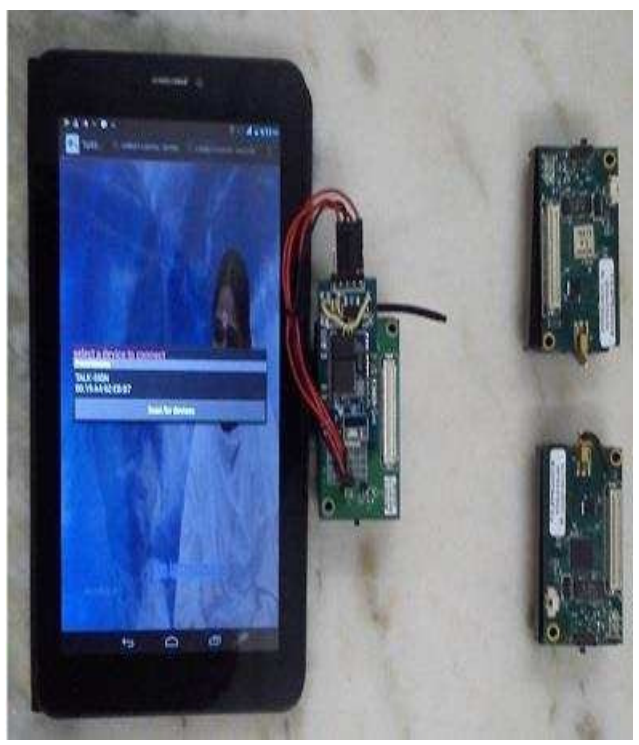

Figure 9. Experimental Setup

The system was tested by implementing it on the corridor of our institution. Three rooms such as one conference hall, one lab and one staff room were instrumented with the device. The person with the user module and an android tab with the application installed are able to recognize the 
rooms accurately. The experiment is repeated with fitting the device in ten other rooms of which some of them are close to each other and others not. The person is able to discover the rooms accurately without any interference from the motes in nearby rooms.

\section{CONCLUSION AND FUTURE WORK}

The RF system using android phone could help blind people with indoor navigation. The working of the system is explained in detail in this document. An installed Android application in his/her phone and the lightweight user module in waist belt will help them to identify locations where they are standing. RF based communication helps them to connect with wall mounted devices easily since RF has an omni directional coverage. Users' phone successfully delivered voice message about the location based on the information received from the wall mounted RF transmitter. For experimentation we used mote but in large scale production we can develop an RF device of less cost.

Future work can be done by adding exact routing information about the user environment. This can be performed by making an indoor routing map of the construction and adding the information into android application, using additional sensors like accelerometers can be used to locate the person and detect obstacles around him. Alert messages can also be given when the user deviates from the exact path and when the system detects obstacles. The other future work includes optimizations in the design of the device and user study covering larger varied proportions of visually impaired persons so as to increase the usability of system as a product.

\section{ACKNOWLEDGEMENT}

We extend our sincere thanks to Mr.Harish kotyian of Reserve Bank of India for all the support and suggestions from his visually challenged perspective.

\section{REFERENCES}

[1] “Assistive technology usage,” http://www.uaa.alaska.edu/dss/information/images/

[2] "Who media center impairment and blindness," http://www.who.int/mediacentre/factsheets/fs282/en/

[3] G. Gayathri, M. Vishnupriya, R. Nandhini, and M. Banupriya, "Smart walk- ing stick for visually impaired," in International Journal of Engineering and Computer Science, 1955.

[4] S. Chumkamon, P. Tuvaphanthaphiphat, and P. Keeratiwintakorn, "A blind navigation system using rfid for indoor environments," in Electrical Engi- neering/Electronics, Computer, Telecommunications and Information Tech- nology, 2008. ECTI-CON 2008. 5th International Conference on, vol. 2, May 2008, pp. 765-768.

[5] Sabarish, "Navigation tool for visually challenged using microcontroller," in International Journal of Engineering and Advanced Technology (IJEAT), vol. 2, April 2013, pp. 1-6.

[6] B. Ding, H. Yuan, X. Zang, and L. Jiang, "The research on blind navigation system based on rfid," inWireless Communications, Networking and Mobile Computing, 2007. WiCom 2007. International Conference on, Sept 2007, pp.2058-2061.

[7] M. V. Kanchan and M. Wankhade, "Visually impaired assistive system," in nternational Journal of Computer Applications, September 2013, pp. 5-10.

[8] M. A. Hersh and J. Ohene-Djan, Eds., Proceedings of the Conference and Workshop on Assistive Technologies for People with Vision and Hearing Impairments: Assistive Technology for All Ages (CVHI-2007), Granada, Spain, 28th - 31th August, 2007, ser. CEUR Workshop Proceedings, vol.415. CEUR-WS.org, 2008.

[9] "Micaz mote," http://www.openautomation.net/

[10] "Blue link silver bluetooth modem," http://www.rhydolabz.com/

[11] "Mda100," http://www.memsic.com/wireless-sensor-networks/MDA100/ 


\section{AUTHORS}

Aswathy V R received her MCA from CHMM College, Kerala, India in July 2012. She is currently pursuing MTech in Wireless Networks and Applications from AmritaVishwaVidyapeetham, Kerala, India. She is currently working on Blind navigation based project and her area of interest includes computer networking and wireless security.

Prof. Sethuraman Rao is an associate professor at Amrita Center for Wireless Networks and Applications, Amrita University, Kollam, Kerala, India. He holds a Masters degree in Computer Science and a Bachelor's degree in Mechanical Engineering from IIT Madras, India. He has over 20 years of international experience in the networking industry having held technical and management positions at Juniper Networks, Alcatel-Lucent and a few startups. His areas of interest include wired and wireless LANs, wireless security, software engineering and network management.

Dilraj N. currently serves as a Research Associate at the Amrita Center for Wireless Networks \& Applications (Amrita WNA), Amritapuri Campus. He completed his M. Tech. in Wireless Networks and Applications, Master of Science in Electronics.
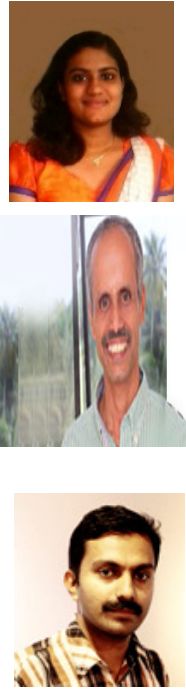